\title{
Mid-Infrarot-Ergebnisse zum Fettsäuremuster der Kuhmilch von Praxisbetrieben
}

\section{Mid-infrared results on fatty acid pattern of milk from dairy farms}

\author{
Andreas Steinwidder ${ }^{1 *}$, Edina Scherzer ${ }^{1,2}$, Werner Zollitsch ${ }^{2}$
}

\begin{abstract}
${ }^{1}$ Höheren Bundeslehr- und Forschungsanstalt für Landwirtschaft HBLFA Raumberg-Gumpenstein, Institut für Biologische Landwirtschaft und Biodiversität der Nutztiere, 8952 Irdning-Donnersbachtal, Österreich

${ }^{2}$ Universität für Bodenkultur Wien (BOKU), Department für nachhaltige Agrarsysteme, Institut für Nutztierwissenschaften, Gregor-MendelStraße 33, 1180 Wien, Österreich

* Korrespondierender Autor: andreas.steinwidder@raumberg-gumpenstein.at
\end{abstract}

Einreichung: 11. September 2019, überarbeitete Einreichung: 19. Februar 2020, Annahme: 11. März 2020

\section{Zusammenfassung}

Die Untersuchung der Milchfettzusammensetzung gewinnt in der Milchbranche an Bedeutung. Zur Bestimmung werden heute, neben den klassischen Analyseverfahren wie der Gaschromatographie (GC), auch kostengünstigere Schnellmethoden angewandt. In der vorliegenden Arbeit sollte die Genauigkeit der über ein Mid-Infrarot-Verfahren (MIR) des „MilkoScan-FT6000-Software-Pakets“ ermittelten Fettsäurekonzentrationen anhand von GC-Fettsäuregehalten evaluiert werden, wobei die Ergebnisse aus der GC-Analyse als Goldstandard angesehen wurden. Dazu wurde auf ausgewählte Proben von Praxisbetrieben bzw. LKW-Tankmilchproben von Milchviehbetrieben einer Kärntner Molkerei (Kärntnermilch) zurückgegriffen. Dreimal jährlich (November 2017, März 2018, Juni 2018) wurden auf fünf Praxisbetrieben (2 biologisch und 3 konventionell wirtschaftende) der Kärntnermilch Liefermilch-Hofmischproben sowie von drei Milchsammeltouren Tankmilchproben (2 biologische und 1 konventionelle) gezogen. Die Milchproben der biologisch wirtschaftenden Betriebe entfielen dabei alle auf Bio-Wiesenmilch-Betriebe. Basierend auf dem GC-Datensatz und einem zusätzlichen umfassenden MIR-Datensatz von Hofmischproben (Januar 2019 und Juni 2019) sollten auch Effekte der Bewirtschaftung (biologisch „Bio-Wiesenmilchprojekt“ bzw. konventionell „gentechnikfrei“) sowie der Saison (Sommer- bzw. Winterfütterungssituation) auf die Milch-Fettsäurekonzentrationen untersucht werden. Da speziell im Bio-Wiesenmilchprojekt der Kärntnermilch eine grünland- und weidebasierte Fütterung angestrebt wird, sollten zusätzlich die Möglichkeiten zur Evaluierung der Rationszusammensetzung über MIRFettsäure-Datensätze beurteilt werden. Es wurden signifikante Unterschiede im Fettsäuremuster zwischen den Wirtschaftsweisen (BioWiesenmilch versus konventionelle gentechnikfreie Milch) und den Saisonen (Sommer- versus Winterfütterungssituation) festgestellt. Die Anteile an mehrfach ungesättigten Fettsäuren (PUFA), konjugierten Linolsäuren (CLA) und Omega-3-Fettsäuren ( $\omega$-3-FA) lagen in den Proben der Bio-Wiesenmilchbetriebe signifikant über jenen der konventionellen Betriebe. Unabhängig von der Wirtschaftsweise lagen in den Sommermilchproben die CLA-, einfach ungesättigten Fettsäuren (MUFA) und ungesättigten Fettsäuren (UFA) höher als in den Wintermilchproben, wobei die saisonalen Effekte bei den Bio-Betrieben stärker ausgeprägt waren als bei den konventionellen Betrieben. Die MIR-Analyseergebnisse korrelierten hinsichtlich der Konzentrationen an UFA bzw. gesättigten Fettsäuren (SFA) sowie an MUFA mit den Ergebnissen der GC-Analytik hoch $(r=0,95)$. Es zeigten sich jedoch systematische Abweichungen, die bei der Ergebnisinterpretation bzw. bei Eichungen zu berücksichtigen sind. Hinsichtlich der Konzentration an PUFA zeigte sich nur ein moderater Zusammenhang $(r=0,57)$ zwischen den Analysemethoden. Außerdem wurde eine große Streuung festgestellt: $95 \%$ der MIR-Ergebnisse für die PUFA lagen zwischen 9,5\% und 40,4\% unter den GC-Ergebnissen. Aus den Ergebnissen der Studie sowie Literaturdaten kann abgeleitet werden, dass aus MIR-Fettsäureergebnissen alleine keine eindeutigen und direkten Rückschlüsse auf die Fütterung (z. B. Weide-, Kraftfutter- oder Maissilage-Rationsanteil) von Kühen gezogen werden können. Die MIR-Ergebnisse könnten jedoch als grobes Screening-Tool für darauf aufbauende Betriebsberatungskonzepte genutzt werden.

Schlagworte: Milch, Fettsäuren, Mid-Infrarot-Spektroskopie, biologisch 


\section{Summary}

The composition of milk fat is gaining importance in the dairy industry. In addition to classical analytical methods - such as gas chromatography $(\mathrm{GC})$ - more time- and cost-effective rapid methods are now available for determining the milk fat composition. In the present study, the accuracy of fatty acid concentrations determined by mid-infrared spectroscopy (MIR) was evaluated with the "MilkoScan FT6000 Software Package". The fatty acid contents determined with the GC were considered as "gold standard". For this purpose, selected milk samples from individual farms or tank milk samples of farms in Carinthia (dairy "Kärntnermilch") were used. Three times a year (November 2017, March 2018, June 2018), delivery milk samples were taken from five farms (2 organic and 3 conventional farms) of Kärntnermilch and from three milk collection rounds (tank milk samples from 2 organic and 1 conventional round). All organic farms followed the production guidelines of "Bio-Wiesenmilch". Based on the GC-dataset and an additional comprehensive MIR data set of farms in Carinthia (January 2019 and June 2019), the effects of the management (organic "Bio-Wiesenmilch" versus conventional "GMO-free") and period (summer versus winter feeding situation) on the milk fatty acid concentrations were investigated. Since the "Bio-Wiesenmilch" production scheme of the Kärntnermilch aims at grassland and pasture-based feeding systems, the possibilities for checking the ration composition via MIR fatty acid data sets had also been evaluated. The proportions of relevant fatty acid groups in milk were significantly different between the production systems (organic versus conventional) and periods (summer versus winter feeding situation). In the milk samples of the organic farms, the proportions of polyunsaturated fatty acids (PUFA), conjugated linoleic acids (CLA) and omega-3-acids ( $\omega$-3-FA) were significantly higher than in those of the conventional farms. Regardless of the production system, the CLA, monounsaturated fatty acids (MUFA) and unsaturated fatty acids (UFA) concentrations in the summer milk samples were higher than those in the winter milk samples. The seasonal effects were more pronounced in the organic system than in the conventional system. The MIR analysis results were highly correlated $(\mathrm{r}=0.95)$ with the results of the GC analysis for the concentrations of UFA, saturated fatty acids (SFA) and MUFA. However, there were systematic deviations that have to be taken into account when interpreting the results. For the PUFA only a moderate correlation $(r=0.57)$ was found between the two methods. Additionally, a substantial variance was found, 95 of the MIR results for PUFA differed between $-9.5 \%$ and $-40.4 \%$ from the GC results. From the results of the study and from the literature it can be derived that MIR fatty acid results alone allow no clear and direct conclusions on the feeding strategy (e. g. dietary proportion of pasture, concentrated feed or maize silage). However, the MIR results could be used as a rough screening tool for farm advisory concepts.

Keywords: milk, fatty acids, mid-infrared spectroscopy, organic

\section{Einleitung}

Die Erfassung der Fettzusammensetzung gewinnt in der Molkereiwirtschaft zunehmend an Bedeutung, da über die Aufnahme von Milchprodukten ein bedeutender Beitrag zur ausgewogenen Fettsäureaufnahme in der menschlichen Ernährung geleistet werden kann. Die Fütterungsbedingungen beeinflussen die Fettsäurezusammensetzung bei Einzelkühen bzw. auf Herdenebene in der Tankmilch deutlich (Ferlay et al., 2008; Hanuš et al., 2018). Außerdem wurden genetische Einflüsse (Rasse) sowie Tier- (Laktationsstadium, Milchleistungsniveau usw.) und Umwelteinflüsse (Höhenlage usw.) auf das Fettsäuremuster beschrieben (Jensen, 2002; Kalač und Samková, 2010; Adamska et al., 2016).

Die Analytik der Fettsäuregehalte (FA) mittels Gaschromatographie (GC) stellt ein standardisiertes, präzises, aber relativ aufwendiges Messverfahren dar. Für die routinemäßige Analytik gewinnen kostengünstigere Schnellmethoden an Bedeutung. Dazu zählt auch die Mid-Infrarot-Spektroskopie (MIR), welche in der Milchverarbeitungsbranche seit Jahrzehnten zur Bestimmung der milchpreisrelevanten Milchinhaltsstoffe (Fett, Eiweiß etc.) verwendet wird. Mengenmäßig dominierende Fettsäuregruppen oder Fettsäuren können durch die MIR-Analyse präzise erhoben werden. Im Vergleich zur GC-Analyse nimmt die Genauigkeit mit abnehmender Fettsäurekonzentration in der Milch ab. Die MIR-Analytik arbeitet in einem Wellenzahlenbereich (400 bis $4000 \mathrm{~cm}^{-1}$ ), in dem chemische Verbindungen besondere Absorptionseigenschaften zeigen. Das dabei erzeugte MIR-Spektrum veranschaulicht diese Absorptionen in verschiedenen Wellenzahlenbereichen. Wie Forschungsergebnisse zeigen, ist die FA-Bestimmung in Milch mittels MIR-Analytik bei mengenmäßig bedeutenden Fettsäuregruppen (z. B. ungesättigten Fettsäuren) bzw. einzelnen Fettsäuren (z. B. C12:0, C14:0) als robust und präzise einzustufen, die Genauigkeit nimmt jedoch mit fallenden Konzentrationen ab (Soyeurt et al., 2006, 2011; De Marchi et al., 2011). Das Fett der Kuhmilch besteht zu etwa $70 \%$ aus gesättigten Fettsäuren (SFA), 25-27\% aus einfach ungesättigten Fettsäuren (MUFA) und 3 bis $5 \%$ mehrfach ungesättigten Fettsäuren (PUFA). 
Ein Ziel der vorliegenden Arbeit war es, die Genauigkeit der mittels MIR ermittelten Fettsäurekonzentrationen im Vergleich zu gaschromatographisch bestimmten Fettsäuregehalten anhand von Betriebs- bzw. LKW-Tankmilchproben zu evaluieren. Des Weiteren sollten anhand dieser Proben sowie eines umfangreichen MIR-Fettsäuredatensatzes Effekte der Wirtschaftsweise (biologisch „Bio-Wiesenmilch“ versus konventionell "gentechnikfrei“) und der Saison (Sommerversus Winterfütterung) auf die Milch-Fettsäurekonzentrationen untersucht werden. Darüber hinaus sollte auch die Nachvollziehbarkeit einer grünland- und weidebasierten Fütterung (Bio-Wiesenmilch) anhand von MIR-FettsäureDatensätzen für Beratungszwecke beurteilt werden.

\section{Material und Methode}

\subsection{Feldproben „GC- und MIR-Fettsäurebestimmung“}

Dreimal jährlich (Wintersaison: 1.-5. Nov. 2017 und 1.-5. März 2018; Sommersaison: 1.-5. Juni 2018) wurden auf fünf Praxisbetrieben der Kärntnermilch (2 biologisch wirtschaftende Betriebe; 3 konventionell wirtschaftende Betriebe) Liefermilch-Mischproben sowie von drei Milchsammelwagentouren Tankmilchproben (3 Regionen, 2 Wirtschaftsweisen; Katschtal (biologisch), Patergassen-Obermillstatt (biologisch), unteres Drautal (konventionell)) gezogen. Die Milchproben der biologisch wirtschaftenden Betriebe entfielen alle auf Bio-Wiesenmilch-Betriebe. Diese müssen spezielle, über die üblichen Richtlinien (VO (EG) Nr. 834/2007; VO (EG) Nr. 889/2008) hinausgehende Vorgaben hinsichtlich Mindestanteilen an Weide- und Grünfutter während der Vegetationsperiode bzw. an konserviertem Grünfutter während der Winterfütterungsperiode sowie eine Begrenzung des Maissilage- und Kraftfutteranteils einhalten (ARGE Biowiesenmilch, o. J.). Die konventionellen Milchproben kamen überwiegend von Silagebetrieben, auf denen - vorwiegend im Winter - meist auch bedeutende Mengen an Maissilage gefüttert werden. Die von den Betrieben bzw. Tankmilchwägen gezogenen Milchproben $(500 \mathrm{ml})$ wurden geschüttelt und dann auf zwei Teilproben zu je $250 \mathrm{ml}$ aufgeteilt. Jeweils eine Probe davon wurde vor der gaschromatographischen Untersuchung an der HBLFA Raumberg-Gumpenstein tiefgefroren. Die zweite Probe wurde unmittelbar nach der Probenziehung zur Bestimmung der Milchinhaltsstoffe und Fettsäuregehalte mittels Mid-Infrarot-Analytik herangezogen.

\subsection{Lieferbetriebsproben „MIR-Fettsäurebestimmung“}

Der umfangreiche Lieferbetriebsdatensatz umfasste MIRErgebnisse von 2101 Proben aus 1065 Betrieben, welche die Kärntnermilch beliefern (Winterbeprobungen im Januar 2019: 1065 Datensätze; Sommerbeprobungen im Juni 2019: 1036 Datensätze). Davon wurden jene Betriebsdatensätze ausgeschieden, für die keine vollständige Winter- plus Sommerbeprobung vorlag. Es wurden auch jene Datensätze biologisch wirtschaftender Betriebe ausgeschieden $(\mathrm{n}=10)$, deren Milch nicht in die Bio-Wiesenmilch-Schiene der Kärntnermilch geliefert wurde. Der für die Auswertung verwendete Datensatz $(n=1966)$ enthielt 466 Datensätze von 233 BioWiesenmilchbetrieben und 1500 Datensätze von 750 konventionell (gentechnikfrei) wirtschaftenden Betrieben.

\subsection{Gaschromatographische Milchfettsäureanalytik}

Die Extraktion des Fettes für die gaschromatographische Fettsäuren-Analyse (Feldprobendatensatz s. Abschn. 2.1) wurde nach der Methode von Folch et al. (1957) mit leichter Modifikation durchgeführt. Anschließend erfolgte die Derivatisierung zu Fettsäuremethylester mit Trimethylsulfoniumhydroxid (DGF, 2006). Die Bestimmung der Einzel-Fettsäuren erfolgte mittels Gaschromatographen (Varian, Modell 3900), ausgestattet mit einem FlammenIonisierungs-Detektor, einem automatischen Injektor, einem Split-Injektor sowie der Säule Supelco SPTM 2380 (100 $\mathrm{m} \times 0,25 \mathrm{~mm} \times 0,2 \mu \mathrm{m}$ Filmdicke). Die Injektionsbzw. Detektionstemperatur betrug 250 bzw. $260{ }^{\circ} \mathrm{C}$. Als Trägergas diente Helium bei konstantem Säulendruck (3,4 bar). Die Säulentemperatur wurde zu Beginn für 1 Minute bei $60^{\circ} \mathrm{C}$ gehalten; dann wurde die Temperatur mit $8{ }^{\circ} \mathrm{C}$ pro Minute bis auf $120^{\circ} \mathrm{C}$ und anschließend mit $1,5^{\circ} \mathrm{C}$ pro Minute bis auf $240{ }^{\circ} \mathrm{C}$ erhöht. Für die PeakIdentifikation wurden ein Standardmix mit 37 unterschiedlichen Fettsäuremethylestern (Supelco Inc.) sowie individuellen Fettsäure-Standards von Sigma Aldrich, Supelco, Matreya und Larodan verwendet. Die Chromatogramme wurden mit der Software Galaxy ${ }^{\bullet}$ Version 1.9 (Varian) aufgezeichnet und ausgewertet. Die Identifizierung der einzelnen Fettsäuren erfolgte anhand der Retentionszeiten, die mithilfe von FAME-Standardsubstanzen ermittelt wurden. Die Standardsubstanzen dienten außerdem zur Kalkulation von Response-Faktoren für die einzelnen Verbindungen. Mithilfe dieser Faktoren wurde der jeweilige prozentuale Anteil der Fläche eines Fettsäuremethylesters an der Gesamtfläche aller FAME berechnet. 
Die Angabe der Ergebnisse erfolgte in \% FAME (g/100 g Gesamt-FA) (Kämmerer, 2009). Die Einzel-Fettsäuren wurden zu folgenden Fettsäuregruppen zusammengefasst: Gesättigte Fettsäuren (SFA): $\sum$ (C4:0, C5:0, C6:0, C7:0, C8:0, C10:0, C11:0, C12:0, C13:0anteiso, C13:0, C14:0, C15:0iso, C15:0anteiso, C15:0, C16:0iso, C16:0, C17:0iso, C17:0anteiso, C17:0, C18:0, C19:0anteiso, C19:0, C20:0, C21:0, C22:0, C23:0, C24:0)

Einfach ungesättigte Fettsäuren (MUFA): $\sum$ (C14:1c9, C15:1c10, C16:1c9, C17:1c10, C18:1t6,9,11, C18:1c9, C18:1c11, C20:1, C22:1c13, C24:1c15)

Mehrfach ungesättigte Fettsäuren (PUFA): $\sum$ (CLA, $\omega-3-F A$, $\omega-6-F A)$

Konjugierte Linolsäure (CLA): C18:2c9t11

Omega-3-Fettsäuren ( $\omega-3-\mathrm{FA}): \sum$ (C18:3c9,12,15, C20:3c11, 14,17, C20:5c5,8,11,14,17, C22:5c7,10,13,16,19, C22:6c4, $7,10,13,16,19)$

Omega-6-Fettsäuren ( $\omega-6-\mathrm{FA}): \sum(\mathrm{C} 18: 2 \mathrm{t} 9,12, \mathrm{C} 18: 2 \mathrm{c} 9$, 12, C18:3c6,9, 12, C20:2c11,14, C20:3c8,11,14, C20:4c5, $8,11,14, \mathrm{C} 22: 2 \mathrm{c} 13,16)$

\subsection{MIR-Fettsäuregruppen-Analyse}

Die Milchproben (Feldproben s. Abschn. 2.1; Lieferbetriebsproben s. Abschn. 2.2) wurden im Labor der Kärntnermilch einer Mid-Infrarot-Schnellanalyse (MIR) mittels MilkoScan FT6000 unterzogen. Dieses Analyseverfahren wurde gewählt, weil dieses Gerät in der Molkerei routinemäßig zum Einsatz kommt. Vor der Untersuchung wurden die Proben auf $40+/-3{ }^{\circ} \mathrm{C}$ erwärmt und mittels MilkoScan FT6000 die Milchinhaltstoffe (Fett, Protein, Laktose, Casein, Harnstoff, Fettsäuregruppen) auf Basis der FTIR-Technik (Fourier Transform Infrared Spectroscopy) bestimmt. Über das MilkoScan-FT6000-Software-Paket werden zusätzlich auch Gehalte zu folgenden Fettsäuregruppen in g/100 ml Milch ausgewiesen: ungesättigte Fettsäuren (UFA), einfach ungesättigte Fettsäuren (MUFA), mehrfach ungesättigte Fettsäuren (PUFA) und gesättigte Fettsäuren (SFA). Details zur Methodik der Fettsäurebestimmung können in FOSS (o. J.) nachgelesen werden. Die Molkerei führt einmal wöchentlich eine Kalibrierung mit den Standards, die von der QSE GmbH (aus Wolnzach) zugesendet werden, durch. QSE liefert immer die Zertifikate der jeweiligen Charge mit. Zusätzlich nimmt die Molkerei monatlich an einem Ringtest zur Standardisierung der Spektren teil. Dadurch wird das Zusammenführen von Spektren verschiedener Instrumente ermöglicht. Außerdem kann durch die Standardisierung ein höheres Maß an Homogenität und
Stabilität durch die monatliche Korrektur in der Vorhersage erreicht werden (EMR und CRA-W, o. J.). Die Gehalte der vier Fettsäuregruppen (in g/100 g Milch angegeben) wurden anschließend auf die Gehalte je $100 \mathrm{~g}$ Fettsäuren (g/100 g Fettsäuren) umgerechnet (z. B. UFA [g/100 g Fettsäure $]=$ UFA [g/100 g Milch]/(SFA + UFA [g/100 g Milch] × 100). Im Gegensatz zur GC-Analyse ergibt die Summe der MIRKonzentrationen der Fettsäuregruppen MUFA und PUFA nicht die Konzentration von UFA, weil die Messwerte direkt aus der Analyse übernommen wurden.

\subsection{Statistische Auswertung}

Mithilfe des Statistikprogramms SAS 9.4 (SAS Institute Inc., Cary, NC, USA) wurden die gaschromatographisch ermittelten Fettsäure-Ergebnisse der konventionellen Milchproben mit den Bio-Wiesenmilch-Proben in einem gemischten Modell mit den fixen Effekten „Bewirtschaftung" (bio vs. kon) und „Saison“ (Wi: Winter vs. So: Sommer) sowie dem zufälligen getesteten Effekt für Betrieb (bzw. Region) innerhalb Bewirtschaftung (FreiheitsgradApproximation ddfm $=\mathrm{kr}$ ) ausgewertet. Die Ergebnisse dazu werden als Least-Square-Means angegeben, zusätzlich werden auch die Residualstandardabweichung $\left(\mathrm{s}_{\mathrm{e}}\right)$ und die P-Werte für die Variablen „Wirtschaftsweise“ und „Saison“ sowie die Wechselwirkung "Wirtschaftsweise $\times$ Saison“ angeführt. Die paarweisen Vergleiche wurden mithilfe des Tukey-Tests durchgeführt, Mittelwerte mit unterschiedlichen Hochbuchstaben weisen in den Ergebnissen auf signifikante Differenzen $(\mathrm{p}<0,05)$ hin.

Der Vergleich der GC-Analyse und MIR-Analyse erfolgte mit dem Statistikprogramm STATGRAPHICS ${ }^{\circledR}$ Centurion XVII (Statpoint Technologies, Inc., The Plains, Virginia, USA) mithilfe des Korrelationskoeffizienten nach Pearson. Nach Held (2010) weist ein Korrelationskoeffizient von über 0,3 auf einen schwachen, über 0,5 auf einen moderaten und über 0,8 auf einen engen Zusammenhang zwischen zwei Variablen hin. Außerdem wurden die durchschnittliche Übereinstimmung der Messwerte sowie die Streuung und Lage der individuellen Messwertdifferenzen mithilfe der „Bland-Altman-Analyse“ analysiert (Grouven et al., 2007). Die Ergebnisse der gaschromatographischen Analysen wurden dabei als „Goldstandard“ angesehen.

Der umfangreiche MIR-Datensatz der Lieferbetriebe (s. Abschn. 2.2) wurde mit dem SAS 9.4-Statistikprogramm (SAS Institute Inc., Cary, NC, USA) mit einem gemischten Modell und den fixen Effekten „Bewirtschaftung" (bio versus kon) und „Saison“ (W: Winter bzw. 
S: Sommer) sowie dem zufälligen getesteten Effekt für Betrieb innerhalb Wirtschaftsweise (Freiheitsgrad-Approximation $\mathrm{ddfm}=\mathrm{kr}$ ) ausgewertet. Die Ergebnisse dazu werden als Least-Square-Means angegeben, zusätzlich werden auch die Residualstandardabweichung (s) und die P-Werte für die Variablen „Wirtschaftsweise“ und „Saison“" sowie die Wechselwirkung "Wirtschaftsweise $\times$ Saison“ angeführt. Die paarweisen Vergleiche wurden mithilfe des Tukey-Tests durchgeführt, Mittelwerte mit unterschiedlichen Hochbuchstaben weisen auf signifikante Differenzen $(\mathrm{p}<0,05)$ hin.

\section{Ergebnisse}

\subsection{Effekte der Wirtschaftsweise und Saison auf GC-Fettsäurekonzentrationen (Feldproben)}

In Tabelle 1 sind die gaschromatographisch ermittelten Ergebnisse ausgewählter Fettsäuregruppen für die Effekte „Wirtschaftsweise“ und „Saison“ bzw. die Wechselwirkung aus Wirtschaftsweise $\times$ Saison dargestellt. Die Anteile an PUFA, CLA und $\omega-3$-FA lagen in den Proben der
Bio-Wiesenmilchbetriebe signifikant über jenen der konventionellen Betriebe. Unabhängig von der Wirtschaftsweise lagen in den Sommermilchproben die CLA-, MUFA- und UFA-Konzentrationen höher als in den Wintermilchproben. Dabei ist zu beachten, dass bei den SFA-, MUFA-, UFA- und CLA-Konzentrationen signifikante Wechselwirkungen zwischen der Wirtschaftsweise und der Saison bestanden: Bei den biologischen Milchproben waren die saisonalen Effekte deutlicher ausgeprägt als bei den konventionellen Proben; die Sommermilchproben der Bio-Betriebe wiesen die höchsten MUFA-, PUFA-, UFAund CLA-Gehalte auf. Zwischen den konventionellen und biologischen Milchproben zeigten sich in den Sommerfütterungsmonaten größere Differenzen im Fettsäuremuster als in der Winterfütterungsperiode.

\subsection{Methodenvergleich (GC-Analytik und MIR-Schnellanalytik)}

Die Ergebnisse in Tabelle 2 sowie in Abbildung 1 zeigen für UFA-, MUFA- und SFA-Konzentrationen hohe Korrelationen $(r=0,95)$ zwischen den Ergebnissen der unterschiedlichen Analysenmethoden. Trotzdem muss im

Tabelle 1. Anteile an ausgewählten Fettsäuregruppen (GC-Analytik bzw. MIR-Schnellanalytik) in der Milch (ausgewählter Praxisbetriebsdatensatz) Table 1. Proportions of important groups of fatty acids (GC-analysis or MIR-analysis) in the milk (selected farms dataset)

\begin{tabular}{|c|c|c|c|c|c|c|c|c|c|c|c|c|}
\hline \multirow[t]{2}{*}{ Fettsäuren $^{1}$} & \multicolumn{2}{|c|}{$\begin{array}{c}\text { Wirtschaftsweise } \\
\text { (W) }\end{array}$} & \multicolumn{2}{|c|}{$\begin{array}{c}\text { Saison } \\
\text { (S) }\end{array}$} & \multicolumn{4}{|c|}{$\begin{array}{c}\text { Saison } \times \text { Wirtschaftsweise } \\
(\mathrm{S} \times \mathrm{W})^{2}\end{array}$} & \multirow[t]{2}{*}{$s_{\mathrm{e}}$} & \multicolumn{3}{|c|}{ P-Werte } \\
\hline & bio & kon & So & Wi & So bio & So kon & Wi bio & Wi kon & & $\mathbf{W}$ & $S$ & $\mathrm{~S} \times \mathrm{W}$ \\
\hline \multicolumn{13}{|l|}{ GC-Analytik } \\
\hline SFA, g/100 g FA & 70,4 & 72,5 & 70,0 & 72,9 & $67,9^{\mathrm{b}}$ & $72,1^{\mathrm{a}}$ & $73,0^{\mathrm{a}}$ & $72,9^{\mathrm{a}}$ & 1,08 & 0,108 & $<0,001$ & $<0,001$ \\
\hline MUFA, g/100 g FA & 25,4 & 23,9 & 26,0 & 23,2 & $27,8^{\mathrm{a}}$ & $24,2^{\mathrm{ab}}$ & $23,0^{\mathrm{b}}$ & $23,5^{\mathrm{b}}$ & 1,02 & 0,233 & $<0,001$ & 0,001 \\
\hline PUFA, g/100 g FA & 4,16 & 3,58 & 3,98 & 3,76 & $4,33^{\mathrm{a}}$ & $3,63^{\mathrm{bc}}$ & $4,00^{\mathrm{ab}}$ & $3,53^{c}$ & 0,28 & 0,003 & 0,088 & 0,364 \\
\hline UFA, g/100 g FA & 29,6 & 27,4 & 30,0 & 27,0 & $32,1^{\mathrm{a}}$ & $27,9^{\mathrm{b}}$ & $27,0^{\mathrm{b}}$ & $27,0^{\mathrm{b}}$ & 1,13 & 0,092 & $<0,001$ & 0,001 \\
\hline CLA, g/100 g FA & 1,11 & 0,75 & 1,07 & 0,79 & $1,33^{\mathrm{a}}$ & $0,82^{b}$ & $0,90^{\mathrm{b}}$ & $0,69^{\mathrm{b}}$ & 0,12 & 0,010 & $<0,001$ & 0,014 \\
\hline iso $\mathrm{FA}, \mathrm{g} / 100 \mathrm{~g} \mathrm{FA}$ & 1,89 & 1,85 & 1,86 & 1,87 & 1,91 & 1,81 & 1,86 & 1,88 & 0,11 & 0,701 & 0,886 & 0,241 \\
\hline$\omega-3-F A, g / 100 \mathrm{~g}$ FA & 1,12 & 0,81 & 0,91 & 1,03 & $1,05^{\mathrm{ab}}$ & $0,76^{\mathrm{b}}$ & $1,19^{\mathrm{a}}$ & $0,86^{\mathrm{ab}}$ & 0,17 & 0,043 & 0,143 & 0,754 \\
\hline$\omega-6-F A, g / 100 \mathrm{~g} \mathrm{FA}$ & 1,93 & 2,01 & 2,00 & 1,94 & 1,95 & 2,05 & 1,91 & 1,98 & 0,11 & 0,538 & 0,239 & 0,772 \\
\hline$\omega-6 / \omega-3-V e r h a ̈ l t n i s$ & 1,75 & 2,70 & 2,36 & 2,10 & 1,87 & 2,85 & 1,64 & 2,56 & 0,40 & 0,071 & 0,150 & 0,851 \\
\hline \multicolumn{13}{|l|}{ MIR-Schellanalytik } \\
\hline SFA, g/100 g FA & 67,0 & 69,5 & 66,8 & 69,7 & $64,9^{b}$ & $68,7^{\mathrm{a}}$ & $69,2^{\mathrm{a}}$ & $70,3^{\mathrm{a}}$ & 1,33 & 0,068 & $<0,001$ & 0,029 \\
\hline MUFA, g/100 g FA & 26,9 & 25,3 & 27,2 & 25,0 & $28,8^{\mathrm{a}}$ & $25,6^{\mathrm{b}}$ & $25,0^{\mathrm{b}}$ & $25,0^{\mathrm{b}}$ & 1,00 & 0,075 & $<0,001$ & 0,003 \\
\hline PUFA, g/100 g FA & 3,09 & 2,80 & 3,18 & 2,71 & $3,40^{a}$ & $2,96^{\mathrm{b}}$ & $2,78^{b}$ & $2,64^{\mathrm{b}}$ & 0,29 & 0,001 & 0,002 & 0,245 \\
\hline UFA, g/100 g FA & 33,0 & 30,5 & 33,2 & 30,3 & $35,1^{b}$ & $31,3^{\mathrm{a}}$ & $30,8^{\mathrm{a}}$ & $29,7^{\mathrm{a}}$ & 1,33 & 0,068 & $<0,001$ & 0,029 \\
\hline
\end{tabular}


Mittel mit Differenzen und mit systematischen Abweichungen der Messergebnisse gerechnet werden (Tabelle 1 bzw. Tabelle 3). Bei der MIR-Analytik wurden die UFAKonzentrationen in allen Proben im Vergleich zu den gaschromatographischen Ergebnissen über- und dementsprechend die SFA-Konzentrationen unterschätzt. Entsprechend der Bland-Altman-Analyse lagen in durchschnittlich $95 \%$ der Fälle die Konzentrationen nach der MIR-Analytik bei den UFA um 1,6 bis 4,9 g/100 g FA absolut höher und dementsprechend in der SFA-Konzentration um 1,7 bis 4,7 g/100 g FA tiefer als jene nach der GC-Analytik (Tabelle 3). In der MUFA-Konzentration wurden im Mittel höhere MIR-Werte festgestellt, wobei die Methodendifferenzen bei den MUFA bei abnehmenden MUFA-Gehalten in der Milch zunahmen. $95 \%$ der Daten wichen in den MUFA-Konzentrationen, analysiert mit der MIR-Analytik, im Bereich von 3,2 bis $-0,06 \mathrm{~g} / 100 \mathrm{~g}$ FA von den Ergebnissen der GC-Analytik ab. Bei den PUFA lag demgegenüber der Korrelationskoeffizient mit 0,57 deutlich tiefer (Tabelle 3). Alle ausgewiesenen Werte der MIR-Schnellanalysenmethode waren geringer als jene der GC-Analyse. $95 \%$ der Messwertpaare zeigten eine absolute Differenz zwischen den Methoden von $-0,27$ bis $-1,67 \mathrm{~g} / 100 \mathrm{~g}$ FA bzw. wichen relativ zwischen 9,5 und 40,4\% negativ von den GC-Analysenergebnissen ab.

\subsection{MIR-Fettsäuregehalte der Lieferbetriebsproben bei Winter- bzw. Sommerfütterung}

Die MIR-Ergebnisse zu den Milchinhaltstoffen und der Fettsäurezusammensetzung sind in Tabelle 4 zusammengefasst. Mit Ausnahme von Fett, Milchharnstoff und Laktosegehalt zeigte sich bei allen Parametern eine signifikante Wechselwirkung zwischen Wirtschaftsweise (Bio-Wiesenmilch bzw. konventionell-gentechnikfrei) und Saison (Sommer- bzw. Winterfütterungssituation).
Die Bio-Betriebe lieferten sowohl im Sommer als auch im Winter eine Milch mit geringerem Fett- und Eiweißgehalt, wobei die Differenzen zwischen den Wirtschaftsweisen im Winter deutlicher als im Sommer ausgeprägt waren. Der Milchharnstoffgehalt war bei beiden Wirtschaftsweisen im Sommer etwas höher als im Winter, wobei in der Milch der konventionellen Betriebe im Mittel höhere Harnstoffgehalte festgestellt wurden. Auch bei allen mit der MIR-Analytik ermittelten Fettsäuregruppen-Konzentrationen (UFA, MUFA, PUFA und SFA) waren die Differenzen zwischen den Wirtschaftsweisen in der Sommerfütterungssituation stärker ausgeprägt als bei Winterfütterung. Die Fettsäurezusammensetzung differierte zwischen den Saisonen (Sommer, Winter) bei biologisch wirtschaftenden deutlicher als bei den konventionellen Betrieben. Es zeigte sich bei keinem untersuchten Parameter diesbezüglich jedoch in den Faktorkombinationen $(\mathrm{W} \times \mathrm{S})$ eine Effektumkehr. Für die Milchproben der biologisch wirtschaftenden Betriebe ergaben sich sowohl bei der Sommer- als auch bei der Winterfütterungssituation signifikant geringere Konzentrationen an SFA bzw. signifikant höhere Konzentrationen an UFA als in den entsprechenden konventionellen Untergruppen. In den über die MIR-Schnellmethode ermittelten Konzentrationen an PUFA lagen die Bio-Betriebe sowohl in der Sommer- (3,5 g/100 g FA) als auch in der Winterfütterungssituation (3,3 g) über jenen der konventionellen Betriebe, welche im Mittel bei 3,2 g/100 g FA lagen.

In Abbildung 2 ist die Häufigkeitsverteilung der Fettsäurekonzentrationen für UFA, MUFA und PUFA der biologischen wirtschaftenden Betriebe in der Sommer- und Winterfütterungssituation dargestellt. In der Sommerfütterungssituation lagen jeweils $5 \%$ der Betriebe in der UFA-Konzentration unter $31,1 \mathrm{~g} / 100 \mathrm{~g}$ FA, in der MUFA- Konzentration unter $24,5 \mathrm{~g} / 100 \mathrm{~g}$ FA und in

Tabelle 2. Beziehungen zwischen den Ergebnissen der GC- und MIR-Analytik für die Fettsäuregruppen SFA, MUFA, PUFA und UFA Table 2. Relationship between results from GC- and MIR-analyses for the fatty acid groups SFA, MUFA, PUFA und UFA

\begin{tabular}{|c|c|c|c|c|c|c|c|c|c|}
\hline & \multicolumn{2}{|c|}{ arithm. Mittel ${ }^{1,2}$} & \multicolumn{2}{|c|}{ Standardabw..$^{1,2}$} & \multirow[t]{2}{*}{ lineares Regressionsmodell ${ }^{1,2}$} & \multirow[t]{2}{*}{ P-Wert } & \multirow[t]{2}{*}{$\mathbf{r}$} & \multirow{2}{*}{$\begin{array}{c}\text { Standardfebler } \\
\text { Schätzung }\end{array}$} & \multirow{2}{*}{$\begin{array}{c}\text { Mittlerer } \\
\text { Absolutfehler }\end{array}$} \\
\hline & GC & MIR & $G C$ & MIR & & & & & \\
\hline SFA2 & 72,0 & 68,8 & 2,42 & 2,49 & SFA-GC $=8,50+0,9229 \times$ SFA - MIR & $<0,001$ & 0,95 & 0,78 & 0,66 \\
\hline MUFA2 & 24,2 & 25,7 & 2,32 & 1,83 & MUFA-GC $=-6,85+1,2050 \times$ MUFA - MIR & $<0,001$ & 0,95 & 0,76 & 0,65 \\
\hline PUFA2 & 3,83 & 2,87 & 0,396 & 0,350 & PUFA-GC $=2,00+0,6391 \times$ PUFA - MIR & 0,004 & 0,57 & 0,34 & 0,27 \\
\hline UFA2 & 28,0 & 31,2 & 2,43 & 2,49 & UFA-GC $=-0,81+0,9220 \times$ UFA - MIR & $<0,001$ & 0,95 & 0,82 & 0,71 \\
\hline
\end{tabular}

${ }^{1}$ Angaben in $\mathrm{g} / 100 \mathrm{~g} \mathrm{FA}$

${ }^{2}$ SFA bzw. SFA-GC oder SFA-MIR = gesättigte Fettsäuren, ermittelt mit GC- oder MIR-Analytik; MUFA bzw. MUFA-GC oder MUFA-MIR = einfach ungesättigte Fettsäuren, ermittelt mit GC- oder MIR-Analytik; PUFA bzw. PUFA-GC oder PUFA-MIR = mehrfach ungesättigte Fettsäuren, ermittelt mit GC- oder MIR-Analytik; UFA bzw. UFA-GC oder UFA-MIR = ungesättigte Fettsäuren, ermittelt mit GC- oder MIR-Analytik 


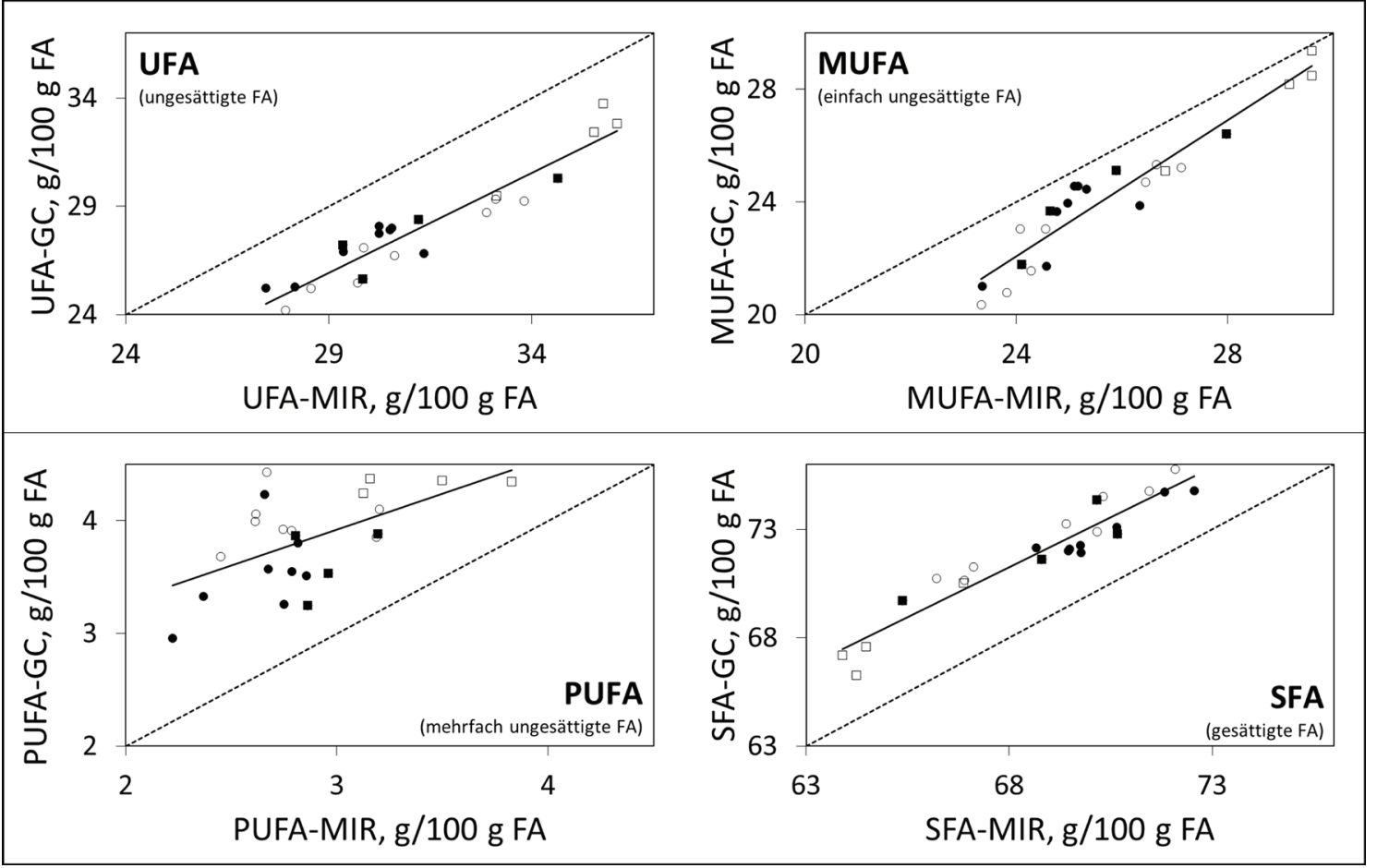

Abbildung 1. Gegenüberstellung der Ergebnisse aus GC- bzw. MIR-Analytik für die Fettsäuregruppen UFA, MUFA, PUFA und SFA (in g/100 g FA; •...konventionell Winterfütterung, ○ ...bio Winterfütterung, •...konventionell Sommerfütterung, $\square$...bio Sommerfütterung) Figure 1. Comparison of results from GC- and MIR-analysis for the fatty acid groups UFA, MUFA, PUFA and SFA (in g/100 g FA; •...conventional winter feeding, o...organic winter feeding, $\cdot . .$. conventional summer feeding, $\square$...organic summer feeding)

der PUFA-Konzentration unter 2,8 g/100 g FA. Würde man in der Sommerfütterungssituation bei jeder der oben angeführten Fettsäuregruppen diesen „5-\%-Grenzwert“ bei den biologisch wirtschaftenden Betrieben anwenden, dann würden $7,7 \%$ der Betriebe zumindest bei einer Fettsäuregruppe den jeweiligen Grenzwert, 3,4 \% der Betriebe zumindest bei zwei Fettsäuregruppen bzw. 1,3\% der Betriebe bei allen drei Fettsäuregruppen (UFA, MUFA und PUFA) den jeweiligen oben angeführten Grenzwert unterschreiten.

\section{Diskussion und Schlussfolgerungen}

\subsection{Effekte der Wirtschaftsweise und der Saison}

Im Fettsäuremuster wurden signifikante Unterschiede zwischen den Wirtschaftsweisen (Bio-Wiesenmilch und konventionelle gentechnikfreie Milch) und den Saisonen (Sommerbzw. Winterfütterungssituation) festgestellt. Es zeigte sich bei den gaschromatographisch ermittelten UFA-, MUFA- und CLA-Konzentrationen eine signifikante Wechselwirkung

Tabelle 3 Bland-Altman-Analyse zu den Ergebnissen der GC- und MIR-Analytik für die Fettsäuregruppen SFA, MUFA, PUFA und UFA Table 3. Bland-Altman analysis of the results from GC- and MIR-analysis for the fatty acid groups SFA, MUFA, PUFA and UFA

\begin{tabular}{|c|c|c|c|c|c|c|}
\hline & $\begin{array}{l}\varnothing \text { Differenz } \\
\text { (GC-MIR) }\end{array}$ & $\begin{array}{c}\text { obere Grenze } \\
\text { Differenz }\end{array}$ & $\begin{array}{c}\text { untere Grenze } \\
\text { Differenz }\end{array}$ & $\begin{array}{c}\emptyset \text { Quotient } \\
\text { (MIR/GC) }\end{array}$ & $\begin{array}{c}\text { oberer Grenze } \\
\text { Quotient }^{3}\end{array}$ & $\begin{array}{c}\text { untere Grenze } \\
\text { Quotient }^{3}\end{array}$ \\
\hline SFA $^{2}$ & $-3,199$ & $-1,658$ & $-4,740$ & 0,956 & 0,977 & 0,934 \\
\hline MUFA $^{2}$ & 1,570 & 3,201 & $-0,060$ & 1,068 & 1,149 & 0,987 \\
\hline PUFA $^{2}$ & $-0,967$ & $-0,267$ & $-1,668$ & 0,750 & 0,905 & 0,596 \\
\hline UFA $^{2}$ & 3,243 & 4,870 & 1,617 & 1,117 & 1,180 & 1,054 \\
\hline
\end{tabular}

${ }^{1}$ Angaben in g/100 g FA

${ }^{2} \mathrm{SFA}=$ gesättigte Fettsäuren, MUFA = einfach ungesättigte Fettsäuren, PUFA = mehrfach ungesättigte Fettsäuren, UFA = ungesättigte Fettsäuren

${ }^{3} 95 \%$ der Ergebnisse liegen zwischen den jeweils angegebenen Werten der oberen und unteren Grenze 


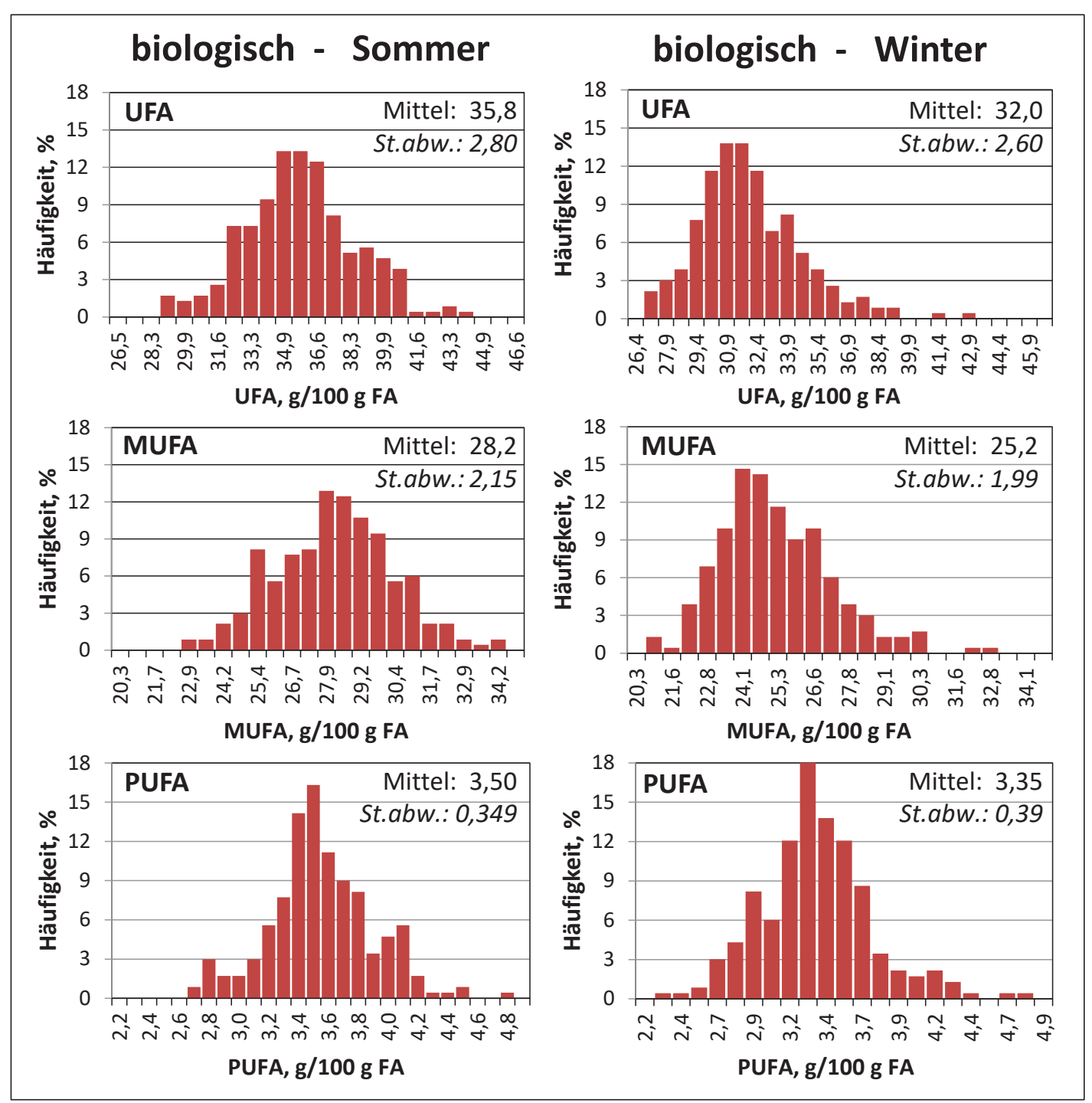

Abbildung 2. Häufigkeitsverteilung der Fettsäurekonzentrationen für UFA, MUFA und PUFA der Milch der biologisch wirtschaftenden Betriebe in der Sommer- (links) und Winterfütterungssituation (rechts) aus der MIR-Analytik

Figure 2. Frequency distribution of fatty acid concentrations for UFA, MUFA and PUFA in the milk of the organic farms in a summer (left) or winter feeding period (right) from MIR-analysis

zwischen den Haupteffekten „Wirtschaftsweise“ und „Saison", wobei diese Wechselwirkung jedoch nicht über eine Effektumkehr in den Untergruppen zustande kam. Die Differenzen im Fettsäuregehalt zwischen der Milch der BioWiesenmilchbetriebe und jener der konventionellen Betriebe waren jedoch im Sommer stärker ausgeprägt als im Winter, und der saisonal bedingte Unterschied in den Gehalten an UFA, MUFA und CLA war bei den Bio-Wiesenmilchproben wesentlich höher als bei den konventionellen Milchproben. Unabhängig von der Güte der MIR-Fettsäureanalytik (s. Abschn. 4.2) zeigten sich diese Wechselwirkungseffekte auch in den MIR-Datensätzen (Feldproben bzw. Lieferbetriebsproben).

Diese Unterschiede im Fettsäuremuster können vorwiegend auf die Rationsparameter zurückgeführt werden. Nach Hanuš et al. (2018) beeinflussen die Fütterung bzw. die Fettsäuregehalte der Ration das Milchfettsäuremuster mit rund $55 \%$ am deutlichsten. Außerdem können aber auch Tier- (Genetik, Laktationsstadium, Milchleistungsniveau usw.) sowie weitere Umwelteinflüsse (bspw. Höhenlage) auftreten (Kalač und Samková, 2010; Jensen, 2002; Adamska et al., 2016). Bio-Wiesenmilchbetriebe müssen in der 
Tabelle 4. Milchinhaltsstoffe und Fettsäurekonzentration, gemessen mit der MIR-Analyse für die biologisch bzw. konventionell wirtschaftenden Lieferbetriebe bei Sommer bzw. Winterfütterung

Table 4. Milk constituents and fatty acid concentrations measured with MIR analysis for the organic or conventional farmers in a summer- or winter-feeding period

Fett, \%

Eiweiß, \%

Laktose, \%

Harnstoff, mg/100 ml

SFA, g/100 g FA

MUFA, g/100 g FA

PUFA, g/100 g FA

UFA, g/100 g FA

\begin{tabular}{|c|c|c|c|c|c|c|c|c|c|c|c|}
\hline \multicolumn{2}{|c|}{$\begin{array}{l}\text { Wirtschaftsweise } \\
\text { (W) }\end{array}$} & \multicolumn{2}{|c|}{$\begin{array}{l}\text { Saison } \\
(\mathbf{S})\end{array}$} & \multicolumn{4}{|c|}{$\begin{array}{c}\text { Saison } \times \text { Wirtschaftsweise } \\
(\mathrm{S} \times \mathrm{W})\end{array}$} & \multirow[t]{2}{*}{$s_{\mathrm{e}}$} & \multicolumn{3}{|c|}{ P-Werte } \\
\hline bio & kon & So & Wi & So bio & So kon & Wi bio & Wi kon & & W & $S$ & $S \times W$ \\
\hline 4,18 & 4,35 & 4,13 & 4,40 & $4,05^{\mathrm{d}}$ & $4,20^{c}$ & $4,31^{\mathrm{b}}$ & $4,50^{\mathrm{a}}$ & 0,23 & $<0,001$ & $<0,001$ & 0,118 \\
\hline 3,31 & 3,47 & 3,33 & 3,45 & $3,27^{\mathrm{d}}$ & $3,40^{\mathrm{b}}$ & $3,35^{c}$ & $3,54^{a}$ & 0,15 & $<0,001$ & $<0,001$ & $<0,001$ \\
\hline 4,84 & 4,86 & 4,87 & 4,83 & $4,86^{\mathrm{b}}$ & $4,88^{a}$ & $4,83^{c}$ & $4,84^{\mathrm{c}}$ & 0,06 & 0,004 & $<0,001$ & 0,053 \\
\hline 22 & 24 & 24 & 22 & $23^{\mathrm{b}}$ & $26^{a}$ & $22^{c}$ & $23^{\mathrm{b}}$ & 5,41 & $<0,001$ & $<0,001$ & 0,282 \\
\hline 66,1 & 67,8 & 65,3 & 68,6 & $64,2^{\mathrm{d}}$ & $66,3^{c}$ & $68,0^{\mathrm{b}}$ & $69,2^{\mathrm{a}}$ & 2,39 & $<0,001$ & $<0,001$ & 0,002 \\
\hline 26,7 & 25,8 & 27,6 & 24,9 & $28,2^{a}$ & $27,0^{\mathrm{b}}$ & $25,2^{c}$ & $24,6^{\mathrm{d}}$ & 1,82 & $<0,001$ & $<0,001$ & 0,008 \\
\hline 3,4 & 3,2 & 3,4 & 3,2 & $3,5^{\mathrm{a}}$ & $3,2^{\mathrm{c}}$ & $3,3^{\mathrm{b}}$ & $3,2^{\mathrm{d}}$ & 0,30 & $<0,001$ & $<0,001$ & 0,021 \\
\hline 33,9 & 32,2 & 34,7 & 31,4 & $35,8^{\mathrm{a}}$ & $33,7^{\mathrm{b}}$ & $32,0^{c}$ & $30,8^{\mathrm{d}}$ & 2,39 & $<0,001$ & $<0,001$ & 0,002 \\
\hline
\end{tabular}

Bewirtschaftung und vor allem in der Fütterung besondere Richtlinien einhalten. Dazu zählt sowohl die Weideverpflichtung in der Vegetationsperiode als auch die Begrenzung von Futtermitteln aus Ackerkulturen (Getreide, Maissilage etc.). Daten von Scherzer (2019) sowie die Ergebnisse von Arbeitskreisbetrieben (BMNT, 2019) lassen auf BioWiesenmilchbetrieben im Durchschnitt einen geringeren Einsatz von Kraftfutter und Maissilage sowie einen höheren Weide- und Grünfutteranteil in der Ration im Vergleich zu konventionellen Betrieben erwarten. Bei weide- oder grünfutterbasierter Fütterung wurden in zahlreichen Versuchen hohe UFA-, MUFA-, PUFA- und CLA- sowie geringe SFAKonzentrationen in der Milch festgestellt (White et al., 2001; Elgersma et al., 2006; Bellof et al., 2013; Kiendler et al, 2019; Scherzer et al., 2020). Mit steigenden Rationsanteilen an Kraftfutter oder Maissilage muss insbesondere mit einem Rückgang der PUFA- und CLA-Konzentrationen in der Milch gerechnet werden (Butler et al., 2008; Schulz et al., 2018). Sowohl bei den konventionellen als auch bei den biologisch wirtschaftenden Betrieben wurden signifikante Saisoneffekte (Sommer- bzw. Winterfütterung) festgestellt. Für beide Wirtschaftsweisen wurden in der Sommerfütterungssituation höhere Gehalte an UFA, MUFA und CLA ermittelt. Zu den signifikanten Wechselwirkungen (Wirtschaftsweise $\times$ Saison) kam es deshalb, weil der Saisoneffekt bei den Bio-Betrieben diesbezüglich deutlicher als bei den konventionellen Betrieben ausgeprägt war. Dies ist ein Hinweis darauf, dass sich bei biologischer Wirtschaftsweise die Sommerfütterungsbedingungen deutlicher von jenen des Winters unterschieden, als das bei den konventionellen Betrieben der Fall ist. Aber auch auf den untersuchten konventionell wirtschaftenden Betrieben muss von beachtenswerten Rationsänderungen im Jahresverlauf (z. B. Maissilageanteil im Sommer geringer; Grünfütterung bzw. Weidehaltung häufiger) ausgegangen werden (Scherzer, 2019).

\subsection{Methodenvergleich}

Nach den Ergebnissen der vorliegenden Arbeit sowie nach Soyeurt et al. $(2006,2011)$ und De Marchi et al. (2011) ist die Fettsäure-Bestimmung mittels Mid-Infrarot-Spektroskopie (MIRS) bei mengenmäßig dominierenden Fettsäuregruppen (z. B. UFA) und auch bei einigen individuellen Fettsäuren (z. B. C12:0; c9-C18:1) als robust und präzise einzustufen, die Genauigkeit nimmt mit abnehmenden Fettsäurekonzentrationen in der Milch ab. Zur Prüfung der Messgenauigkeit unterschiedlicher Messmethoden schlagen Grouven et al. (2007) über den Korrelationskoeffizienten hinaus die Bland-Altman-Analyse als grafische Methode vor. Diese visualisiert die durchschnittliche Übereinstimmung und die Streuung der individuellen Messwertdifferenzen. Ein Korrelationskoeffizient von über 0,3 weist auf einen schwachen, über 0,5 auf einen moderaten und über 0,8 auf einen engen Zusammenhang zwischen zwei Variablen hin (Held, 2010). Entsprechend dem hohen Korrelationskoeffizienten von 0,95 stimmten die MIR-Analyseergebnisse hinsichtlich der Konzentrationen an ungesättigten (UFA) bzw. gesättigten Fettsäuren (SFA) mit den jeweiligen Ergebnissen der GC-Analysen sehr gut überein. Es zeigte sich jedoch eine systematische Abweichung, bei Anwendung der MIR-Analyse wurden die UFA-Konzentrationen bei allen Proben und in jedem 
Konzentrationsbereich in vergleichbarem Ausmaß leicht überschätzt. Auch für die MUFA-Konzentrationen zeigte sich ein hoher Korrelationskoeffizient von 0,95. Es wurden aber auch hier systematische Abweichungen zwischen den MIR- und GC-Ergebnissen festgestellt, wobei diese im niedrigeren Konzentrationsbereich stärker als im hohen Konzentrationsbereich ausgeprägt waren. Demgegenüber zeigte sich für die in der Milch in geringeren Konzentrationen vorkommenden PUFA mit einem Korrelationskoeffizienten von 0,57 nur ein moderater Zusammenhang zwischen den Ergebnissen der unterschiedlichen Analysemethoden. Bei MIR-Analytik wurden bei allen Proben geringere PUFA-Konzentrationen als bei der GC-Analyse ausgewiesen. Darüber hinaus wurden auch größere Streuungen festgestellt, entsprechend der Bland-Altman-Analyse wichen $95 \%$ der MIR-Ergebnisse zwischen 9,5\% und $40,4 \%$ negativ von den GC-Ergebnissen ab. Die vorliegenden Ergebnisse decken sich mit Literaturangaben (Soyeurt et al., 2006, 2011; Rutten et al., 2009; De Marchi et al., 2011), wonach die Genauigkeit und Robustheit der MIR-Fettsäurebestimmung bei Fettsäuren bzw. Fettsäuregruppen, die in geringeren Konzentrationen in der Milch vorkommen, möglicherweise bedingt durch die verwendete Geräte-Software derzeit noch begrenzt ist. In diesem Fall erlauben die Ergebnisse nur eine grobe Zuordnung hoher und niedriger Fettsäurekonzentrationen in der Milch.

\subsection{Potenziale der MIR-Fettsäureergebnisse zur Evaluierung der Fütterung}

Informationen zur Zusammensetzung der Futterrationen auf Milchviehbetrieben gewinnen in der Milchbranche an Bedeutung. Beispielsweise fordern Bio-Verbände bzw. Gütesiegel-Programme aus unterschiedlichen Gründen (Tierwohl, Produktqualität, Erwartungen der Konsumenten etc.) in der Vegetationsperiode eine Weide- bzw. Grünfutteraufnahme bzw. generell eine Begrenzung des Kraftfutter- und Ackerfuttereinsatzes (Arge Biowiesenmilch, o. J.; Bio-Austria, 2019). Die Mid-Infrarot-Spektroskopie ist derzeit in der Molkereiwirtschaft die Methode der Wahl zur routinemäßigen Bestimmung der HauptMilchinhaltsstoffe (Laktose, Fett, Eiweiß etc.) in Liefermilchhofproben. Wie die Ergebnisse der Literatur (Soyeurt et al., 2006, 2011; De Marchi et al., 2011) sowie der vorliegenden Arbeit zeigen, können mithilfe der MIR-Analytik darüber hinaus auch Fettsäuregehalte in der Milch, jedoch mit unterschiedlicher Genauigkeit, abgeschätzt werden. Da der Milchfettsäuregehalt wiederum hauptsächlich von der Fütterung beeinflusst wird, besteht Interesse an der zusätzlichen Nutzung der MIR-Fettsäureergebnisse zur Evaluierung der Rationsgestaltung (z. B. grünland- und weidebasierte Fütterung etc.).

Wie die Ergebnisse der vorliegenden Arbeit zeigen, können über die MilkoScan FT6000 ermittelten MIRFettsäuregehalte die Konzentration an ungesättigten bzw. gesättigten Fettsäuren (UFA bzw. SFA) sowie an einfach ungesättigten Fettsäuren (MUFA) in der Milch sehr gut abgeschätzt werden, wobei jedoch bestehende systematische Abweichungen zu berücksichtigen sind. Demgegenüber sind die ausgewiesenen PUFA-Konzentrationen, aufgrund der nur moderaten Methodenübereinstimmung $(r=0,57)$ sowie der deutlichen und auch ungerichteten Streuung nur sehr eingeschränkt zur Fütterungsevaluierung nutzbar. Dies ist im Zusammenhang mit der Evaluierung der Fütterung mithilfe des MIR-Fettsäuremusters der Milch insofern bedeutend, da mit steigenden Rationsanteilen stärkereicher Futtermittel oder Maissilage vor allem mit einem Rückgang der PUFA-Konzentrationen in der Milch zu rechnen ist (Butler et al., 2008; Schulz et al., 2018). Bei weide- oder grünfutterbasierter Fütterung können in der Milch hohe MUFA- und PUFA- und somit geringere SFA-Konzentrationen erwartet werden (White et al., 2001; Elgersma et al., 2006; Bellof et al., 2013; Kiendler et al, 2019; Scherzer et al., 2020). Zu beachten ist dabei jedoch auch, dass sich Tier-, Rations- bzw. weitere Umweltfaktoren nicht immer einheitlich auf die Fettsäurekonzentrationen innerhalb der Fettsäuregruppen (UFA, MUFA, PUFA) auswirken. Beispielsweise kann durch das Verfüttern von Ölsaaten das Fettsäuremuster deutlich beeinflusst werden. Bei Verfütterung von Sojabohnen wurde ein Anstieg der PUFA, CLA, C18:0, C18:2 und C18:3 und eine Abnahme an C16:0 und C18:1 in der Milch festgestellt (Grummer, 1991), bei Einsatz von vollfetten Rapssamen oder Rapsöl stieg der MUFA-Gehalt an und ging der SFA-Gehalt im Vergleich zur Kontrollgruppe zurück (Hoffmann et al., 2016).

Die Ergebnisse der vorliegenden Arbeit lassen daher den Schluss zu, dass aus alleinigen MIR-Ergebnissen derzeit keine eindeutigen und direkten Rückschlüsse auf die Rationszusammensetzung (z. B. Weide-, Kraftfutter- oder Maissilage-Rationsanteil) gezogen werden können. Die Vergleiche der beiden Analysemethoden müssten von einer größeren Anzahl an Betrieben und über längere Zeit hinweg vorliegen. Innerhalb vergleichbarer Betriebsund Saisongruppen können jedoch niedrige UFAund MUFA-Konzentrationen (bedingt auch niedrige 
PUFA-Konzentrationen) bei MIR-Fettsäureanalytik auf eine eingeschränkte weide- oder grünfutterbasierte Fütterung hinweisen. Da hohe UFA- und MUFA-Konzentrationen in der Milch beispielsweise aber auch durch Verfütterung ölhaltiger pflanzlicher Ergänzungsfuttermittel auftreten können, weisen demgegenüber hohe UFA- und MUFA-Konzentrationen nicht zwangsläufig auch auf eine weide- oder grünfutterbetonte Fütterung hin.

\section{Schlussfolgerungen}

- Durch die Fütterung wird das Fettsäuremuster der Milch wesentlich beeinflusst. Sowohl bei konventioneller als auch biologischer Wirtschaftsweise zeigten sich signifikante Differenzen in der Fettsäurekonzentration zwischen der Sommer- und Winterfütterungssituation, wobei diese bei biologischer Wirtschaftsweise stärker als bei konventioneller ausgeprägt waren. Im Vergleich zu den konventionellen Betrieben wies die Milch der biologisch wirtschaftenden Betriebe höhere

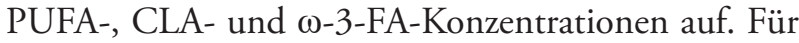
beide Wirtschaftsweisen waren in den Sommermilchproben die CLA-, MUFA- und UFA-Konzentrationen höher als in den Wintermilchproben.

- Über die MilkoScan-FT6000-ermittelten MIR-Fettsäuregehalte kann die Konzentration an ungesättigten bzw. gesättigten Fettsäuren (UFA bzw. SFA) sowie an einfach ungesättigten Fettsäuren (MUFA) in der Milch gut abgeschätzt werden. Die bestehenden geringen systematischen Abweichungen der MIR-Ergebnisse müssen bei der Interpretation beachtet werden.

- Die ausgewiesenen MIR-PUFA-Tankmilchergebnisse erlauben im Vergleich zur GC-Analyse aufgrund der nur moderaten Korrelation und großen Streuung eine sehr grobe Identifikation hoher und niedriger PUFAKonzentrationen in Tankmilchproben von Betrieben. Die Genauigkeit der MIR-Fettsäurebestimmung bei der Fettsäuregruppe PUFA ist aufgrund des Vorkommens in niedrigerer Konzentration durch das Gerät MilkoScan FT6000 bzw. die Geräte-Software begrenzt.

- Aus alleinigen MIR-Fettsäureergebnissen können keine eindeutigen und direkten Rückschlüsse auf die Fütterung (z. B. Weide-, Kraftfutter- oder Maissilage-Rationsanteil) gezogen werden. Unter Berücksichtigung von Saisoneffekten (Sommer, Winter) sowie Daten vergleichbarer Betriebsgruppen könnten jedoch, bei starken Abweichungen im Fettsäuregehalt der Milch, spezielle
Beratungsaktivitäten umgesetzt werden. Beispielsweise könnten in der Sommerfütterungssituation, bei niedrigen Konzentrationen an UFA und MUFA (und eingeschränkt auch bei sehr niedrigen PUFA-Konzentrationen), Maßnahmen zur Optimierung der Weide- und Grünfutteraufnahme ergriffen werden.

\section{Literatur}

Adamska, A., Rutkowska, J. und W. Przybylski (2016): Comparison of fatty acid composition of milk from Simmental and Polish Holstein-Friesian cows in different production seasons. Annals of Animal Science 16, 1211-1225.

Arge Biowiesenmilch (o. J.): https://www.biowiesenmilch. at. Abgerufen am 9. September 2019.

Bellof, G., Ferber, P. und E. Schmidt (2013): Zur Fettsäurezusammensetzung der Kuhmilch in Abhängigkeit von Weidehaltung sowie konventioneller oder ökologischer Wirtschaftsweise. 12. Wissenschaftstagung Ökologischer Landbau Bonn, 504-507.

Bio-Austria (2019): BIO AUSTRIA-Produktionsrichtlinien, März 2019. Hrsg.: BIO AUSTRIA - Verein zur Förderung des Biologischen Landbaus, Linz, $128 \mathrm{~S}$. https:/www.bio-austria.at/app/uploads/BA_Richtlinien_März_2019.pdf. Abgerufen am 9. 9. 2019.

BMNT (Bundesministerium für Nachhaltigkeit und Tourismus, Hrsg.) (2019): Milchproduktion 2018. Ergebnisse und Konsequenzen der Betriebszweigauswertung aus den Arbeitskreisen in Österreich. Bericht BMNT, 38 S.

Butler, G., Nielsen, J.H., Slots, T., Seal, C., Eyre, M.D., Sanderson, R. und C. Leifert (2008): Fatty acid and fat-soluble antioxidant concentrations in milk from high- and low-input conventional and organic systems: seasonal variation. Journal of the Science of Food and Agriculture 88, 1431-1441.

DGF (Hrsg.), (2006): Methode C-VI 11 (98) - Fettsäurenmethylesther (TMSH Methode). In: DGF-Einheitsmethoden: Deutsche Einheitsmethoden zur Untersuchung von Fetten, Fettprodukten, Tensiden und verwandten Stoffen. Wissenschaftliche Verlagsgesellschaft mbH, 2. Aufl., Stuttgart.

De Marchi, M., Penasa, M., Cecchinato, A., Mele, M., Secchiari, P. und G. Bittante (2011): Effectiveness of mid-infrared spectroscopy to predict fatty acid composition of Brown Swiss bovine milk. Animal 5, 16531658. 
De Marchi, M., Toffanin, V., Cassandro, M. und M. Penasa (2014): Invited review: Mid-infrared spectroscopy as phenotyping tool for milk traits. Journal of Dairy Science 97, 1171-1186.

Elgersma, A., Tamminga, S. und G. Ellen (2006): Modifying milk composition through forage - Review. Animal Feed Science and Technology 131, 207-225.

EMR und CRA-W (o. J.): Anleitung für die Untersuchung von Standardisierungsproben. Centre Wallon de Recherches Agronomiques, 5030 Gembloux (Belgique), $2 S$.

Ferrlay, A., Agabriel, C., Sibra, C., Journal, C., Martin, B. und Y. Chilliard (2008): Tank milk variability in fatty acids according to farm feeding and husbandry practices in a French semi-mountain area. Dairy Science Technology 88, 193-215.

Folch, J., Lees, M. und G.H.S. Stanley (1957): A simple method for the isolation and purification of total lipids from animal tissues. Journal of Biological Chemistry 226, 497-509.

FOSS (o. J.): MilkoScan ${ }^{\mathrm{TM}} 7$ RM / FT+ / FT 6000 - Fatty Acid Prediction models. FOSS Application Note 0064 / Rev. 6, Part No. 01026556.

Grouven, U., Bender, R., Ziegler, A. und S. Lange (2007): Vergleich von Messmethoden. Deutsche medizinische Wochenschrift 132, e69-e73.

Grummer, R.R. (1991): Effect of feed on the composition of milk fat. Journal of Dairy Science 74, 3244-3257.

Hanuš, O., Samková, E., Krížová, L., Hasonová, L. und R. Kala (2018): Role of fatty acids in milk fat and the influence of selected factors on their variability - A Review. Molecules 23, 1636.

Held, U. (2010): Tücken von Korrelationen: Die Korrelationskoeffizienten von Pearson und Spearman. Swiss Medical Forum 10, 652-653.

Hoffmann, A., Görlich, S., Steingass, H., Terry, H., Schollenberger, M., Hartung, K. und R. Mosenthin (2016): Milk production and milk fatty acids in dairy cows fed crushed rapeseed or rapeseed oil. Livestock Science 190, 31-34.

Jensen, R.G. (2002): The composition of bovine milk lipids: January 1995 to December 2000. Journal of Dairy Science 85, 295-350.

Kalač, P. und E. Samková (2010): The effects of feeding various forages on fatty acid composition of bovine milk fat: A review. Czech Journal of Animal Science 55, 521-537. Kiendler, S., Gruber, L., Terler, G., Velik, M., Eingang, D., Schauer, A. und M. Royer (2019): Einfluss des
Konservierungsverfahrens von Wiesenfutter auf Futterwert, Futteraufnahme und Milchleistung. 46. Viehwirtschaftliche Fachtagung, 10-11. April 2019, Bericht HBLFA Raumberg-Gumpenstein, 97-109.

Kämmerer, S. (2009): Entwicklung und Anwendung eines NIRS-basierten Routineverfahrens zur Analyse der relativen und absoluten Fettsäurezusammensetzung in Rind- und Schweinefleisch sowie die Untersuchung von Kandidatengenen für den bovinen Fettstoffwechsel (Diss). Friedrich-Schiller-Universität, Jena.

Rutten, M.J.M., Bovenhuis, H., Hettinga, K.A., van Valenberg, H.J.F. und J.A.M. van Arendonk (2009): Predicting bovine milk fat composition using infrared spectroscopy based on milk samples collected in winter and summer. Journal of Dairy Science 92, 6202-6209.

Scherzer, E. (2019): Einfluss der Fütterung von Kühen auf das Fettsäuremuster der Milch - Versuchs- und Praxisergebnisse. Masterarbeit, Universität für Bodenkultur, $104 \mathrm{~S}$.

Scherzer, E., Steinwidder, A., Starz, W., Rohrer, H., Pfister, R., Velik, M. und W. Zollitsch (2020): Einfluss von Vollweide- oder Grassilagefütterung von Kühen auf das Fettsäuremuster der Milch. Züchtungskunde (im Druck).

Schulz, F., Westreicher-Kristen, E., Molkentin, J., Knappstein, K. und A. Susenbeth (2018): Effect of replacing maize silage with red clover silage in the diet on milk fatty acid composition in cows. Journal of Dairy Science 101, 1-12.

Soyeurt, H., Dehareng, F., Gengler, N., McParland, S., Wall, E., Berry, D.P., Coffey, M. und P. Dardenne (2011): Mid-infrared prediction of bovine milk fatty acids across multiple breeds, production systems, and countries. Journal of Dairy Science 94, 1657-1667.

Soyeurt, H., Dardenne, P., Dehareng, F., Lognay, G., Veselko, D., Marlier, M., Bertozzi, C., Mayeres, P. und N. Gengler (2006): Estimating fatty acid content in cow milk using mid-infrared spectrometry. Journal of Dairy Science 89, 3690-3695.

Verordnung (EG) Nr. 834/2007 des Rates vom 28. Juni 2007 über die ökologische/biologische Produktion und die Kennzeichnung von ökologischen/biologischen Erzeugnissen und zur Aufhebung der Verordnung (EWG) Nr. 2092/91 (ABl. L 189 vom 20.7.2007).

Verordnung (EG) Nr. 889/2008 der Kommission vom 5. September 2008 mit Durchführungsvorschriften zur Verordnung (EG) Nr. 834/2007 des Rates über die ökologische/biologische Produktion und die Kennzeichnung von ökologischen/biologischen Erzeugnissen 
hinsichtlich der ökologischen/biologischen Produktion, Kennzeichnung und Kontrolle (ABl. L 250 vom 18.9.2008).

White, S.L., Bertrand, J.A., Wade, M.R., Washburn, S.P., Green, J.T. und T.C. Jenkins (2001): Comparison of fatty acid content of milk from Jersey and Holstein cows consuming pasture or a total mixed ration. Journal of Dairy Science 84, 2295-2301. 